\title{
El espacio urbano. Un lugar de interacción social.
}

\author{
The urban space. A place of social interaction.
}

Eduardo Sousa-González ${ }^{1}$

Desde la evidencia histórica, es posible visualizar que hasta nuestros días en la esfera que circunscribe a lo urbano (ciudades, metrópolis y demás), es claro que estas agrupaciones de ciudadanos, aunque han adquirido una importancia significativa no sólo en virtud del incremento sostenido en el crecimiento numérico de sus pobladores; sino también, en la concentración infraestructural, de equipamientos, de las actividades secundarias, de servicios y de los adelantos tecnológicos propios de la época, no representan, en su mayoría, formas de relaciones humanas equilibradas que adoptar, ni mucho menos se han destacado por generar procesos sociales vinculados a la igualdad, la justicia y la equidad social (Sousa, E. 2008).

Ya desde las ciudades griegas y romanas (polis-civitas) donde existía una asociación de habitantes-poder en un espacio físico determinado ( $u r b s$ ), como un simbolismo de centralidad, se aprecia en mayor o menor grado $\mathrm{y}$ según las diferentes zonas, regiones, estados y municipios del sur global y el mundo en general, no sólo la existencia y la dispersión de estos fenómenos urbanos; sino también, el incremento desmedido de lo que ahora en la sobremodernidad, podemos asociar a por lo menos tres conceptos negativos que están vinculados a estas concentraciones sui géneris de ciudadanos:

i. La crisis de urbanidad, en la que se engloban numerosas condicionales difícilmente controladas en los espacios urbanos, lo cual ha generado y motivado, entre otros, diversas formas contradictorias de segregación social, de hacinamiento, de pobreza extrema, de indolencia social, de la pérdida identitaria, del abandono de la visión que propicia la comprensión del Otro y otras muchas distopías perniciosas.

ii. La crisis espacial, evidentemente enlazada al suelo urbano: a sus diferentes usos, a los deficientes procesos evolutivos, que están asociados a la manera en que se ejecuta la planeación expansiva de orientación periférica de los usos del suelo, a su inequitativa dotación infraestructural y de los equipamientos básicos requeridos por los habitantes, a la falta de dosificación y compatibilización adecuada de los usos del suelo, y su evidente cosificación como mercancía con valor de uso y valor de cambio $^{2}$; incluso Arendt, H. considera al suelo urbano como un producto artificial de la "variedad

\footnotetext{
${ }^{1}$ Mexicano, Doctor en Asuntos Urbanos; profesor-investigador en la División de Postgrado de la Facultad de Arquitectura de la Universidad Autónoma de Nuevo León. Miembro del Sistema Nacional de Investigadores SNI, reconocido en el nivel 2. Miembro de la Academia Mexicana de las Ciencias AMC. eduardo.sousagn@uanl.edu.mx

${ }^{2}$ Aristóteles (Aristóteles: 1837) fue el primero en mencionar esta clasificación: "Toda mercancía tiene dos usos, que le pertenecen esencialmente, sin pertenecerle; sin embargo, del mismo modo: uno es especial a la cosa, el otro no lo es (uso directo y uso de cambio). Marx, K. lo menciona como "...la capacidad de todos productos naturales e industriales de servir a la subsistencia del hombre, recibe la denominación particular de valor de uso; la capacidad que tienen de trocarse unos por otros se la llama valor de cambio (Marx, K.: 1984).
} 
interminable de cosas cuya suma total constituye el artificio humano" (Arendt, H. 2002: 23, 157).

iii. La crisis de gobernabilidad, considerada por Ziccardi, A. (1995:104) como como la capacidad de gobernar, en donde se incorpora todo aquello que surge del campo de las relaciones sociales, del ejercicio de la democracia, del ejercicio de la pluralidad, de las formas de participación ciudadana y representaciones sociales, de la legitimidad que deben generar las acciones gubernamentales. Lo cierto es que esta crisis de gobernabilidad se ha contextualizado por una pérdida generalizada de confianza en las instituciones del Estado; la corrupción institucionalizada ha permeado en múltiples regiones del mundo y en amplias esferas no solamente del poder gubernamental: federal, estatal y municipal; también ha corroído una parte del sector social, sobre todo aquella vinculación perniciosa que se da entre el funcionario-político con los grupos económicamente dominantes.

Como se ha mencionado, nada es nuevo, lo intimidante de este proceso evolutivo actual en las ciudades, es el grado de impulso y diversificación, que estas crisis mencionadas han alcanzado en la contemporaneidad; en este contexto, es posible visualizar en términos generales cuatro circunstancias histórico-sociales propuestas en otras investigaciones (Sousa, E.:2008; 2015), asociadas a estas crisis mencionadas anteriormente; las cuales en mayor o menor grado se relacionan a concentraciones urbanas de países en desarrollo o de "subdesarrollo intermedio" como los nombra Di Filippo (1998:14) similares a México. Dichos procesos están identificados con:

Primero, la vida en sociedad independientemente de la matriz social a la que pertenezca el individuo, requiere de un proceso de aculturación como conditio sine qua non, que le permita participar en las diversas interacciones entre pobladores con diferentes características socioeconómicas, costumbres, identidad, y otras.
Segundo, lo relativo al funcionamiento económico de la ciudad que acusa una marcada división del trabajo, generando explotación en favor de la producción de mercancías industriales, de servicios, de conocimiento, incluso del suelo, con fines de acumulación primaria de capital y por ende, antagonismos de clase derivados de la antinomia histórica humana: la explotación del hombre por el hombre.

Tercero, se refiere a la clase gobernante la cual administra los recursos generados por la sociedad en una forma que pareciera ser la menos adecuada: sin un consenso social real, en muchos casos con objetivos electorales y en otros tantos, favoreciendo a los grupos económicamente dominantes. Además, en tanto administrador normativo del espacio urbano los gobiernos locales y en general el Estado, han cedido ante las demandas generadas en torno a éstos: en unos casos permitiendo la urbanización de áreas ecológicas y protegidas, muchas de ellas propicias para las recargas acuíferas; en otros consintiendo la incorporación al desarrollo urbano de áreas urbanas sin vocación para esto, e incluso gestionando la construcción de infraestructura en monumentos identitarios y muchas irregularidades más, donde están involucrados en claro contubernio: los grupos económicamente dominantes, así como la clase política de élite.

Cuarto, de esta manera, las dependencias ligadas con el ejercicio de la planeación, en su compromiso y obligación de ordenador del espacio urbano-metropolitano se han utilizado, en numerosos casos, procesos de planeación urbana con teorías parciales e importadas, incluso instrumentos operativos decimonónicos, los cuales carecen de una visión real y continuada de largo plazo, y lo que es más serio, sin un proyecto futuro de ciudad consensuado socialmente. En efecto, se ha utilizado una forma de planeación que se pudiera denominar de "contingencia" (Sousa, E.: 2007, 2008, 2015): aquella que cambia lo importante por lo urgente, lo importante por lo electoral, lo importante por la permanencia en el poder, o lo importante por la corrupción; ejercida por lo 
que pudiéramos llamar funcionarios gubernamentales contingenciales, ocasionando, en muchos casos, las características de metropolización que ahora padecemos ${ }^{1}$.

En este contexto, hasta el día de hoy, las ciudades continúan con sus procesos evolucionistas lineales, escalando cada vez con mayor aceleramiento las diferentes etapas de metropolización ; así, son múltiples las variables involucradas que dan pie a la rapidez del crecimiento expansivo de orientación periférica en una ciudad metropolitana, siendo el incremento en la velocidad del movimiento ascendente en el proceso de la vida urbana del lugar, uno de los factores más significativos y reveladores que identifican este aceleramiento expansivo en los procesos de cambio de una ciudad a una metrópoli, el cual está vinculado a lo que se ha identificado como algo distintivo en una concentración urbana de características metropolitanas y posteriormente, en el proceso mismo de su consolidación; nos referimos precisamente al aumento generalizado en este factor mencionado anteriormente como "la velocidad de movimiento ascendente...," el cual, desde esta perspectiva de investigación, tiene una relación de correspondencia biunívoca con: el incremento relativo en el número de habitantes y su densidad (hab/k2), la modificación de las distancias de traslado origen-destino, la accesibilidad a los sistemas de transporte privado y público y el espacio público traducido en la estructura vial; esto se representa en el diagrama 1 (cfr.)

\section{Diagrama 1. Proceso iterativo de metropolización basado en la velocidad del movimiento urbano.}

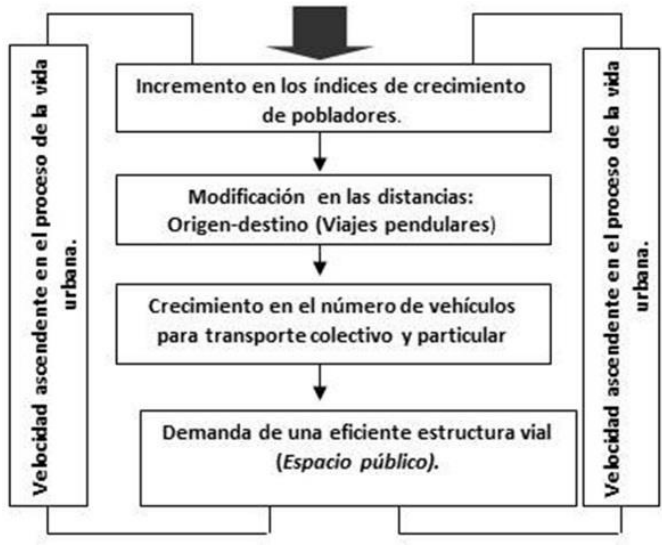

Fuente: Sousa, E: 2008

Desde esta perspectiva relacionada con los procesos urbanos en movimiento asendente, los profesores Francisco José Cuberos-Gallardo y Ibán Díaz-Parra, del Centro de Investigação e Estudos de Sociologia Instituto Universitário de Lisboa (CIES-IUL) y del Departamento de Geografía Humana de la Universidad de Sevilla, España respectivamente; abordan una temática vinculada al barrio del Abasto (Buenos Aires), el cual supone un caso ejemplar de disputa por la centralidad en Buenos Aires, en el que se superponen los proyectos de recualificación urbana orientados al consumo de clase media y al turismo, con la ocupación de viviendas por inmigrantes pobres. La exposición del caso se fundamenta en la combinación de una contextualización de los procesos urbanísticos y sociales desarrollados en el sector con una etnografía que sigue el caso de un edificio de vecinos ocupado por inmigrantes. El trabajo concluye advirtiendo sobre el riesgo de despolitización de una exaltación acrítica de las resistencias cotidianas cuando carecen de una dimensión pública.

\footnotetext{
1 "Funcionario contingencial o urbanista de contingencia, es un concepto que ha sido propuesto Sousa, E. (2007:43), alude a aquellos servidores públicos improvisados, que están incrustados en las dependencias municipales o estatales vinculadas con la planificación del desarrollo urbano y sus derivados (agencias y otras dependencias), los cuales tienen una visión decimonónica y limitada del futuro, además de serias deficiencias de orden teórico-metodológico de aplicación procesal en la operación de la planeación urbana; ya que su formación improvisada en el tema urbano, radica en una visión, en el mejor de los casos, parcial del sitio, lograda a través de su tiempo de adherencia en las diferentes estructuras gubernamentales, mediante un clásico empirismo "fragmentado" (Lefebvre, H. 1980: 53-83): sería algo así como mencionar, que algunos pudiesen tener 20 años de experiencia en
} los problemas de la ciudad, pero... en el mejor de los casos, es la experiencia de un año repetida 20 veces" (Sousa, E.2008). 
En el caso de la profesora Carmen de Tomás Medina del Departamento de Urbanística y Ordenación del Territorio de la escuela Técnica Superior de Arquitectura: Universidad de Sevilla, España; se aborda un tema que vincula a la música de las Cantigas con las ordenanzas de trazado de la ciudad medieval Alfonsina, donde se propone como objeto, descifrar las ordenanzas de trazado que guiaron la construcción de las ciudades alfonsinas y demostrar, mediante el análisis urbanístico de tres de ellas y el estudio de la sabiduría clásica redescubierta en la época, la relación existente entre las reglas de trazado de una ciudad y las reglas de composición musical. Poniendo de manifiesto que las leyes aritméticas y geométricas utilizadas para la composición de la música del Códice de Las Cantigas pudieron ser las mismas que se utilizaron para diseñar el patrón urbano regular.

Brisa Violeta Carrasco Gallegos y Yanely Consuelo Estrada Santoyo, de la Facultad de Geografía de la Universidad Autónoma del Estado de México y El Colegio de Sonora respectivamente, profundizan sobre los productores de suelo urbanizable, teniendo como objetivo de investigación evidenciar la morfología urbana de Hermosillo, Sonora, México, a partir de hacer un análisis histórico descriptivo que permita identificar las zonas urbanas, así como sus consecuencias. La originalidad del trabajo se centra en la falta de estudios que aborden la problemática desde la dimensión urbana para el caso, así la capacidad de demostrar que los megaproyectos urbanos han carecido de una verdadera posibilidad para el desarrollo y por ende, han generado la inserción de otras problemáticas en torno a la ciudad.

Por otro lado, Mario Guadalupe González Pérez del Centro Universitario de Tonalá, de la Universidad de Guadalajara, México, investiga sobre Movilidad cotidiana y habitar periurbano en el área metropolitana de Guadalajara, Jalisco; donde a través del análisis descriptivo y la observación participante se expone la relación entre los costos y beneficios sociales que han presentado recientemente los residentes periurbanos del área metropolitana de Guadalajara, para satisfacer sus necesidades de movilidad y posibilitar con ello, el acceso a sus fuentes de trabajo, servicios de salud, entretenimiento y otros. Los resultados según el autor, sugieren que es necesario un re-pensamiento en materia de planificación de infraestructura y medios de transporte urbano, donde se fundamente el derecho a moverse en la ciudad y hacia la ciudad, mediante una articulación entre accesibilidad y movilidad, donde la primera genere las condiciones de la segunda.

En el caso de los investigadores Rafael de Jesús Huacuz Elías y Rubí del Rocío Vázquez Cruz, profesores de Universidad Michoacana de San Nicolás de Hidalgo, Morelia, Michoacán y de la Facultad de Arquitectura, Universidad de Guanajuato, Guanajuato; analizan el proceso de metropolización en Querétaro entre los años 1990-2010, aplicando el enfoque del análisis territorial, el estudio se centra en el proceso de metropolización el cual ha destacado por lo acelerado que se ha expandido en su superficie de urbanización a través del tiempo. La metodología según los autores, busca estandarizar la medición del fenómeno con el objeto de comparar los resultados entre las ciudades mexicanas. En este caso, la investigación muestra la manera en que un territorio de escala metropolitana se va cubriendo de urbanizaciones y como puede explorarse comparativamente entre las décadas 1990-2010.

Los profesores Jesús Quintana, Arturo Ojeda y Jesús F. García, del Departamento de Ingeniería Civil y Minas, de la Universidad de Sonora, México; muestran los factores que explican el valor del suelo explorando el caso de Hermosillo, Sonora, México; indicando que el análisis parte de una base de datos, geográficamente referenciada, con 427 mediciones de valores del suelo y sus características inmobiliarias, además de, indicadores del Instituto Nacional de Estadística y Geografía, mediciones del Organismo Operador Municipal Agua de Hermosillo y datos publicados por la Secretaría de Seguridad Pública del Estado de Sonora. Datos que se integran en un sistema 
de información geográfica, que permiten establecer modelos de precios hedónicos, que se obtienen utilizando análisis estadísticos y geoestadísticos multivariados, resueltos con la técnica de regresión lineal múltiple y la regresión geográficamente ponderada. Los resultados indican una importante asociación positiva del valor del suelo con el nivel de ingreso, el consumo de agua y la educación; y en este caso, la accesibilidad y la criminalidad en la ciudad parecen ser características que no aportan valor al suelo urbano habitacional.

El tema de la arquitectura como disciplina clave para el estudio del hábitat rural en climas áridos y (semi) desérticos, analizando el caso de Mendoza, Argentina, es abordado por los profesores Virginia Miranda Gassull y Matías Esteves de la Facultad de Ingeniería en la Carrera de Arquitectura -Uncuyo- (Catedra de Vivienda Social) Mendoza, Argentina y del CONICET, Argentina, respectivamente; mencionando que el objetivo es procurar una base de procedimientos metodológicos de abordaje del hábitat rural de zonas no irrigadas, basadas en la inclusión de diversos componentes que inciden en la disposición y análisis arquitectónico. La metodología empleada es la comparación de dos trabajos doctorales realizados en la misma área (provincia de MendozaArgentina). Subrayando que el resultado señala un diseño metodológico basado en la relación naturaleza-cultura y una segunda propuesta deriva de la relación entre el hábitat-territorio según la organización social de base. Se puede destacar que el uso de metodologías cualitativas desde la participación de la población local como actores claves en la construcción del hábitat y el territorio aporta a la comprensión del hábitat y la vivienda como un fenómeno complejo y no solo como objetos materiales.

Reynaldo G. Presmanes y Alberto Álvarez Vallejo adscritos a äDOS ARQUITECTOS, en Cuba y el Centro de Investigación de Arquitectura y Diseño, Universidad Autónoma del Estado de México, México; abordan el tema del Paisaje de la arquitectura. Mirada monista del ambiente, donde el objetivo fue fundamentar el concepto de paisaje de la arquitectura, el cual se ubicó en el contexto de la crisis disciplinaria. La fundamentación, mencionan los autores, se estableció como método de investigación y se soportó teóricamente sobre la epistemología ambiental, el constructivismo, la teoría del diseño y de la arquitectura. Esta labor terminó con la obtención de un concepto de paisaje de la arquitectura de naturaleza epistemológica basado sobre la idea de cultura. $\mathrm{Al}$ respecto, se concluyó identificando al monismo como orden pensamiento emergente, en los estudios del paisaje, a partir de la complejidad y explicando cómo se hace posible a partir del concepto de paisaje de la arquitectura.

En el caso de Pilar Bielma, Arquitecta investigadora en el Instituto Argentino de Investigaciones de las Zonas Áridas (IADIZA) Mendoza, Argentina; nos presenta una reseña del libro del arquitecto Rafael Llácer Pantión (2015). Fachadas marítimas de Cádiz. Colección cuadernos de Paisaje. Granada, Entorno gráfico ediciones. Bielma menciona que el trabajo aquí considerado recoge un conjunto de miradas que han confluido en la ciudad de Cádiz y sus bordes con el mar, distintas aproximaciones a la ciudad que han sabido alimentarse unas de otras en el seno de un proyecto de excelencia. Con él, el grupo humano multidisciplinar que lo acompaña ha querido materializar el trabajo realizado sobre un objeto de estudio concreto -las fronteras entre mar y urbe de un casco histórico profundamente significativo como el de Cádiz-.

El Dr. Jorge Omar García Escamilla profesor Titular de la Facultad de Arquitectura de la Universidad Autónoma de Nuevo León, nos comparte la reseña del libro Espacios Públicos Inclusivos (2017), de los profesores: Nora Livia Rivera Herrera, María Teresa Ledezma Elizondo y Diego Sánchez González; donde el Dr. García menciona que a lo largo de sus trece capítulos este libro presenta para el lector, ya sea profesional especialista de los estudios urbanos, estudiante o simplemente interesado en la forma en la que se configura, se desarrolla y se entiende el espacio en el que vivimos, una serie de perspectivas 
renovadoras y un importante ejercicio de toma de conciencia que contribuye a la creación del conocimiento y a plantear nuevas interrogantes sobre el espacio urbano que día con día construimos entre todos los actores sociales, buscando propiciar un cambio positivo, que incentive un modelo de ciudad más inclusivo, vivible y sostenible para nuestro futuro.

Como comentario final, los miembros del equipo que conforman la Revista CONTEXTO revista de la Facultad de Arquitectura de la Universidad Autónoma de Nuevo León, estamos plenamente convencidos que el investigador y el lector común interesado en la diversa temática expuesta, encontraran a través de las páginas que componen este proyecto editorial, un tópico con una visión interdisciplinar, de actualidad e internacional; enfocada en diferentes esferas del quehacer investigativo; en donde, investigadores de alta calificación, han colaborado con sus trabajos personales o grupales en la generación de este número. Seguramente los artículos que se incluyen aquí serán no solamente de interés, sino también de utilidad concreta en futuras investigaciones, complaciendo al investigador y al lector más exigente.

Finalmente, es necesario reconocer que lo expuesto a través de este número de CONTEXTO, es un esfuerzo continuado a lo largo de muchos años por mantener una calidad de excelencia editorial, que ha sido compartido por autores, pares de evaluadores, el Comité Editorial, la Coordinadora Editorial, el equipo de apoyo y por supuesto, las autoridades institucionales que forman la Facultad de Arquitectura y la Universidad Autónoma de Nuevo León

\section{Bibliografía:}

Arendt, Hanna. (2002). La condición humana. España: Paidos. 21-349.
Filippo Di, Armando. (1998). La visión centro periferia hoy. Revista de Comisión económica para América latina CEPAL. Número extraordinario; pp. 1-14

Lefebvre, Henry. (1980). La revolución urbana, Alianza, Madrid

Marx, Carlos. (1984). Miseria de la filosofía. México, Siglo XXI; pp. 21

Sousa, E. (2007). El área metropolitana de Monterrey. Análisis y propuesta de lineamientos metodológicos para la planeación. México; Colección Trayectorias, UANL.

Sousa, E. (2008). El espacio urbano y su transformación metropolitana en la sobremodernidad. El caso Monterrey, N.L. México. Nóesis. Revista de Ciencias Sociales y Humanidades, Vol. 17, Núm. 34, agosto-diciembre, 2008, pp. 168-203 Universidad Autónoma de Ciudad Juárez, México.

Sousa, E. (2010). Espacios urbanos en la contemporaneidad. Argumentos teóricos para la generación de políticas públicas metropolitanas. México, Universidad autónoma de Nuevo León.

Sousa, E. (2010a). De la ciudad a la metrópoli. Una interpretación teórica del fenómeno expansivo ligado a la vivienda, la vulnerabilidad y la pobreza. En revista INVI ; vol.25, $N^{\circ} 69$; pp. 19-102. Universidad de Chile.

Sousa, E. (2015). De la ciudad a la metrópoli. Nuevas realidades territoriales. Chile, Universidad del Bío-Bío.

Ziccardi, A. (1995). La tarea de gobernar. Gobiernos locales y demandas ciudadanas. México, M. A. Porrúa 